\title{
FRAMED MODULI SPACES AND TUPLES OF OPERATORS
}

\author{
STANISLAV FEDOTOV
}

\begin{abstract}
In this work we address the classical problem of classifying tuples of linear operators and linear functions on a finite dimensional vector space up to base change. Having adopted for the situation considered a construction of framed moduli spaces of quivers, we develop an explicit classification of tuples belonging to a Zariski open subset. For such tuples we provide a finite family of normal forms and a procedure allowing to determine whether two tuples are equivalent.
\end{abstract}

\section{INTRODUCTION}

A quiver $Q$ is a diagram of arrows, determined by two finite sets $Q_{0}$ (the set of "vertices") and $Q_{1}$ (the set of "arrows") with two maps $h, t: Q_{1} \rightarrow Q_{0}$ which indicate the vertices at the head and tail of each arrow. A representation $W$ of $Q$ is a collection of (probably infinite dimensional) $\mathbb{k}$-vector spaces $W_{i}$, for each $i \in Q_{0}$, together with linear maps $W_{a}: W_{t a} \rightarrow W_{h a}$, for each $a \in Q_{1}$. The dimension vector $\alpha \in \mathbb{Z}^{Q_{0}}$ of such a representation is given by $\alpha_{i}=\operatorname{dim}_{\mathbb{k}} W_{i}$. A morphism $\psi: W \rightarrow U$ of representations consists of linear maps $\psi_{i}: W_{i} \rightarrow U_{i}$, for each $i \in Q_{0}$, such that $\psi_{h a} W_{a}=U_{a} \psi_{t a}$, for each $a \in Q_{1}$. It is an isomorphism if and only if each $\psi_{i}$ is.

Having chosen vector spaces $W_{i}$ of dimension $\alpha_{i}$, the isomorphism classes of representations of $Q$ with dimension vector $\alpha$ are in natural one-to-one correspondence with the orbits of the group

$$
G L(\alpha):=\prod_{i \in Q_{0}} G L\left(W_{i}\right)
$$

in the representation space

$$
\operatorname{Rep}(Q, \alpha):=\bigoplus_{a \in Q_{1}} \operatorname{Hom}\left(W_{t a}, W_{h a}\right) .
$$

The action is given by $(g \cdot W)_{a}=g_{h a} W_{a} g_{t a}^{-1}$, where $g=\left(g_{i}\right)_{i \in Q_{0}} \in G L(\alpha)$. Note that the one-parameter subgroup $\Delta=\{(t E, \ldots, t E)\}$ acts trivially.

Quivers provide a convenient interpretation of many classical problems of linear algebra. The one we are particularly interested in is classification of tuples of $q$ linear operators and $k$ linear functions on an $m$-dimensional vector space. In the language of quivers this is equivalent to classification of $(m, 1)$-dimensional representations of $L_{q, k}$, where by $L_{q, k}$ we denote the quiver with two vertices, $q$ loops in the first vertex and $k$ more arrows going from the first vertex to the second one. This problem is known to be wild even for $q=2$ and $k=0$, that is no hope remains to write down a complete list of isomorphism classes of representations or even to obtain an algorithm determining whether two given representations are isomorphic. In fact, representation theory of the quiver $L_{2}:=L_{2,0}$ is proved to be undecidable, see [1] and [6] for a rigorous formulation and a proof of this result.

However, for $\alpha=(m, 1)$ it is possible to explicitly classify representations belonging to a Zariski open subset of $\operatorname{Rep}\left(L_{q, k}, \alpha\right)$. The idea comes from the study of stable framed representations.

2010 Mathematics Subject Classification. Primary 14D22; Secondary 16G10, 16G20. 
Let $Q$ be a quiver and $\alpha$ be a dimension vector. Fix an additional dimension vector $\zeta$ and consider the space $\operatorname{Rep}(Q, \alpha, \zeta):=\operatorname{Rep}(Q, \alpha) \oplus \bigoplus_{i \in Q_{0}} \operatorname{Hom}_{\mathbb{k}}\left(\mathbb{k}^{\alpha_{i}}, \mathbb{K}^{\zeta_{i}}\right)$. Its elements are said to be framed representations of $Q$. Define a $G L(\alpha)$-action on $\operatorname{Rep}(Q, \alpha, \zeta)$ by $g \cdot\left(M,\left(f_{i}\right)_{i \in Q_{0}}\right)=\left(g \cdot M,\left(f_{i} g_{i}^{-1}\right)_{i \in Q_{0}}\right)$. A framed representation $\left(M,\left(f_{i}\right)_{i \in Q_{0}}\right)$ is called stable if there is no nonzero subrepresentation $N$ of $M$ with $M_{i} \subseteq \operatorname{ker} f_{i}$, for all $i \in Q_{0}$. Denote by $\operatorname{Rep}^{s}(Q, \alpha, \zeta)$ the set of stable framed representations. It is known (see, for example, [8, Theorem 2.3]), that the subset $\operatorname{Rep}^{s}(Q, \alpha, \zeta)$ admits a geometric quotient, i.e., a morphism to an algebraic variety $\mathcal{M}^{s}(Q, \alpha, \zeta):=\operatorname{Rep}^{s}(Q, \alpha, \zeta) / / G L(\alpha)$ whose fibers coincide with $G L(\alpha)$-orbits. Moreover, for quivers without oriented cycles M. Reineke proved [8, Proposition 3.9] that the quotient space $\mathcal{M}^{s}(Q, \alpha, \zeta)$ is isomorphic to a Grassmannian of subrepresentations of a certain injective representation of $Q$. In the general case the quotient is not projective and may not be realized as a Grassmannian of subrepresentations. However, some geometric structure may be revealed by projecting $\mathcal{M}^{s}(Q, \alpha, \zeta)$ to the categorical quotient $\operatorname{Rep}(Q, \alpha, \zeta) / / \operatorname{GL}(\alpha)$ and studying fibers of this projection, see [2] and [3] for details.

From [7, Proposition 0.9] it follows that the quotient morphism $\operatorname{Rep}^{s}(Q, \alpha, \zeta) \rightarrow \mathcal{M}^{s}(Q, \alpha, \zeta)$ is a principal fiber bundle. It remains a problem, however, to explicitly describe a finite (and possibly minimal) trivializing covering of the quotient. We construct such a covering using $J$-skeleta of framed representations, a concept that is a version of the one introduced by $\mathrm{K}$. Bongartz and B. HuisgenZimmermann for representations of finite dimensional algebras (see, for example, [4]) adopted and partially simplified to fit our setup. Namely, we show (Theorem 3.3) that $\operatorname{Rep}^{s}(Q, \alpha, \zeta)=\bigcup_{\mathfrak{S}} X(\mathfrak{S})$, where $X(\mathfrak{S})$ are open subsets parameterized by $J$-skeleta $\mathfrak{S}$ such that $X(\mathfrak{S}) \cong \operatorname{GL}(\alpha) \times \mathbb{A}^{N}$, for some positive integer $N$, and the restriction of the quotient map to $X(\mathfrak{S})$ is the projection onto the second factor.

Framed representations admit another useful interpretation. Consider a new quiver $Q^{\zeta}$ with $Q_{0}^{\zeta}=$ $Q_{0} \cup\{\infty\}$, the arrow of $Q^{\zeta}$ being those of $Q$ together with $\zeta_{i}$ arrows from $i\left(i \in Q_{0}\right)$ to $\infty$. We also extend the dimension vector $\alpha$ to $\alpha^{\zeta}$, setting $\alpha_{i}^{\zeta}=\alpha_{i}$ for $i=1, \ldots, n$ and $\alpha_{\infty}^{\zeta}=1$. It is easy to show that $\operatorname{Rep}(Q, \alpha, \zeta)$ may be identified with $\operatorname{Rep}\left(Q^{\zeta}, \alpha^{\zeta}\right)$.

Clearly $\operatorname{Rep}\left(L_{q, k},(m, 1)\right)$ is the same as $\operatorname{Rep}\left(L_{q}^{(k)}, m^{(k)}\right)$, i.e., as $\operatorname{Rep}\left(L_{q}, m, k\right)$. So, there is a Zariski open subset $\operatorname{Rep}^{s}\left(L_{q, k},(m, 1)\right)$ of $\operatorname{Rep}\left(L_{q, k},(m, 1)\right)$ where a complete classification of representations is possible. Translating our definition of stable pairs into the language of linear algebra, we may say that $\operatorname{Rep}^{s}\left(L_{q, k},(m, 1)\right)$ consists of such tuples $\left(\varphi_{1}, \ldots, \varphi_{q}, f_{1}, \ldots, f_{k}\right) \in\left(\operatorname{End}_{\mathbb{k}}\left(\mathbb{k}^{m}\right)\right)^{q} \oplus$ $\left(\left(\mathbb{k}^{m}\right)^{*}\right)^{k}$ that no common proper nonzero invariant subspace of $\varphi_{i}$ lies in the common kernel of all $f_{j}$. In this paper we show how an explicit classification may be obtained in this setup over an arbitrary field $\mathbb{k}$.

Section 1 is devoted to exploring a generalized version of the construction introduced in [8]. In Section 2 we define $J$-skeleta of framed representations and show their existence. In Section 3 we prove that the quotient may be embedded as a locally closed subset in a product of ordinary Grassmannians and construct the above mentioned trivializing covering of $\mathcal{M}^{s}(Q, \alpha, \zeta)$. Furthermore, we provide a finite family of normal forms for each stable pair and an algorithm allowing to determine whether two stable framed representations are isomorphic. In Section 4 we give a series of examples illustrating how this technique works.

The author thanks his supervisor I. Arzhantsev for useful discussions. 


\section{Stable framed Representations}

Let $Q$ be a quiver with $n$ vertices, $\alpha$ and $\zeta$ be two dimension vectors. Choose a vector space $V=$ $\bigoplus_{i \in Q_{0}} V_{i}$ with $\operatorname{dim} V_{i}=\zeta_{i}$, for $i \in Q_{0}$. Elements of $\operatorname{Rep}(Q, \alpha, \zeta)$ may be viewed as pairs $(M, f)$, where $M$ is a representation of $Q$ and $f=\left(f_{i}: M_{i} \rightarrow V_{i}\right)_{i \in Q_{0}}$ is a map of graded vector spaces.

Recall that a path in $Q$ is a formal product of arrows $a_{1} \cdot \ldots \cdot a_{k}$ such that $t\left(a_{i}\right)=h\left(a_{i+1}\right)$, for all $i=1, \ldots, k-1$, or a symbol $e_{i}$ with $i \in Q_{0}$. For a path $\tau=a_{1} \cdot \ldots \cdot a_{k}$ we set $t(\tau)=t\left(a_{k}\right)$ and $h(\tau)=h\left(a_{1}\right)$. We also put $h\left(e_{i}\right)=t\left(e_{i}\right)=i$. There is an obvious way to multiply successive paths: if $h(\tau)=t(\sigma)$, the product $\sigma \cdot \tau$ is defined as the concatenation of these paths. All $e_{i}$ are treated as paths of zero length, that is $e_{i}^{2}=e_{i}$, for all $i \in Q_{0}$, and $\tau e_{t(\tau)}=e_{h(\tau)} \tau$, for every path $\tau$.

For each $i \in Q_{0}$ denote by $I_{i}$ the following representation of $Q$. Set

$$
\left(I_{i}\right)_{j}=(\operatorname{span}\{\tau \mid \tau: j \rightsquigarrow i \text { is a path in } Q\})^{*},
$$

where " $\tau: j \rightsquigarrow i$ " means that $\tau$ starts in the $j$-th vertex and ends in the $i$-th one and $(\cdot)^{*}$ stands for the dual vector space; in this case $\left(\left(I_{i}\right)_{a: k \rightarrow l} f\right)(\tau)=f(\tau a)$, where $\tau: l \rightsquigarrow i$. This may be rewritten in a more convenient way using the elements in $\left(I_{i}\right)_{j}$ dual to paths. Namely, to each path $\tau: j \rightsquigarrow i$ in $Q$ we associate an element $\tau^{*}$ in $\left(I_{i}\right)_{j}$ such that for every $\sigma: j \rightsquigarrow i$ we have

$$
\tau^{*}(\sigma)=\left\{\begin{array}{l}
1, \text { if } \tau=\sigma, \\
0, \text { otherwise. }
\end{array}\right.
$$

Observe that elements of $\left(I_{i}\right)_{j}$ may be written as (probably infinite) formal series in $\tau^{*}$, for $\tau: i \rightsquigarrow j$. In this notation the maps $\left(I_{i}\right)_{a}, a \in Q_{1}$, are as follows:

$$
\left(I_{i}\right)_{a}\left(\tau^{*}\right)=\left\{\begin{array}{l}
\lambda^{*}, \text { if } \tau=\lambda a, \\
0, \text { otherwise }
\end{array}\right.
$$

Consider the representation $J:=\bigoplus_{i \in Q_{0}} I_{i} \otimes_{\mathbb{k}} V_{i}$. Notice that as a $\mathbb{k}$-linear space

$$
J_{i}=e_{i} J \cong \prod_{j \in Q_{0}}\left(I_{j}\right)_{i} \otimes_{\mathbb{k}} V_{j} \cong \prod_{j \in Q_{0}} \prod_{\tau: i \rightsquigarrow j} V_{j} \cong \prod_{\tau: i \rightsquigarrow j} V_{j} .
$$

Sometimes it is convenient to label each component $V_{j}$ by the corresponding path $\tau$ writing $J_{i} \cong$ $\prod_{\tau: i \rightsquigarrow j} V_{j}^{(\tau)}$.

Given a point $(M, f) \in \operatorname{Rep}(Q, \alpha, \zeta)$ we define a map $\Phi_{(M, f)}=\left(\varphi_{i}\right)_{i \in Q_{0}}: M \rightarrow J$ by the following rule:

$$
\varphi_{i}=\prod_{\tau: i \rightsquigarrow j} f_{j} \tau: M_{i} \rightarrow \prod_{\tau: i \rightsquigarrow j} V_{j}
$$

where $\tau(x):=M_{a_{1}} \ldots M_{a_{k}}(x)$ for $x \in M_{i}$ and $\tau=a_{1} \ldots a_{k}$.

The following lemma is straightforward.

Lemma 1.1. The map $\Phi_{(M, f)}$ is a morphism of representations of $Q$.

Proposition 1.2. The subspace $\operatorname{ker} \Phi_{(M, f)}=\oplus_{i \in Q_{0}} \operatorname{ker} \varphi_{i}$ is the maximal $\mathbb{k} Q$-submodule of $M$ contained in $\operatorname{ker} f$.

Proof. It follows from Lemma 1.1 that $\operatorname{ker} \Phi_{(M, f)}$ is a $\mathbb{k} Q$-submodule of $M$. One also easily observes that $\operatorname{ker} \Phi_{(M, f)} \subseteq \operatorname{ker} f$. Now, let $U$ be a $\mathbb{k} Q$-submodule of $M$ contained in $\operatorname{ker} f$. For each $\tau: i \rightsquigarrow j$ we then have $\tau U_{i}=\tau e_{i} U=\tau U=e_{j} \tau U \subseteq U_{j}$. This implies that $f_{j}(\tau \cdot x)=0$, for all $x \in U, j \in Q_{0}$ and for all paths $\tau$, i. e. $U \subseteq \operatorname{ker} \Phi_{(M, f)}$. 
Corollary 1.3. The map $\Phi_{(M, f)}: M \rightarrow J$ is injective if and only if the pair $(M, f)$ is stable.

This observation is crucial for the construction. Associated to the maps $\varphi_{i}$ are (probably infinite) matrices with rows $f_{i q} \tau$, where $\tau: j \rightsquigarrow i, q \in\left\{1, \ldots, \zeta_{i}\right\}$, and $f_{i q}$ stands for the $q$-th row of the matrix of $f_{i}$. The map is injective if and only if one of $\alpha_{i} \times \alpha_{i}$ minors of its matrix is nonzero, i.e., for some $\tau_{1}, \ldots, \tau_{\alpha_{i}}$ and $q_{1}, \ldots, q_{\alpha_{i}}$, we have

$$
\operatorname{det}\left(\begin{array}{c}
f_{i q_{1}} \tau_{1} \\
f_{i q_{2}} \tau_{2} \\
\vdots \\
f_{i q_{\alpha_{i}}} \tau_{\alpha_{i}}
\end{array}\right) \neq 0
$$

Therefore, a pair $(M, f)$ is stable if and only if for each $i \in Q_{0}$ there is a set of numbers $q_{1}^{(i)}, \ldots, q_{\alpha_{i}}^{(i)}$ and a set of distinct paths $\tau_{1}^{(i)}, \ldots, \tau_{\alpha_{i}}^{(i)}$ with

$$
D_{\left(\tau_{1}^{(1)}, \ldots, \tau_{\alpha_{1}}^{(1)}, \ldots ; \tau_{1}^{(n)}, \ldots, \tau_{\alpha_{n}}^{(n)}\right)}^{\left(q_{1}^{(1)}, \ldots, q^{(1)} ; \ldots ;(n)\right.}\left(=\operatorname{det}\left(\begin{array}{c}
f_{i q_{1}^{(1)} \tau_{1}^{(1)}} \\
f_{i q_{2}^{(1)} \tau_{2}^{(1)}}^{(n)} \\
\vdots \\
f_{i q_{\alpha_{1}}^{(1)} \tau_{\alpha_{1}}^{(1)}}
\end{array}\right) \cdot \ldots \cdot \operatorname{det}\left(\begin{array}{c}
f_{i q_{1}^{(n)}} \tau_{1}^{(n)} \\
f_{i q_{2}^{(n)} \tau_{2}^{(n)}} \\
\vdots \\
f_{i q_{\alpha_{n}}^{(n)} \tau_{\alpha_{n}}^{(n)}}
\end{array}\right) \neq 0\right.
$$

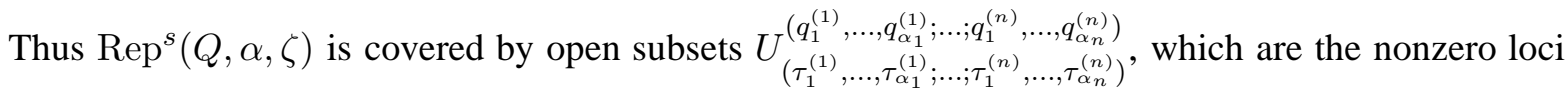
of the corresponding $D_{\left(\tau_{1}^{(1)}, \ldots, \tau_{\alpha_{1}}^{(1)} ; \ldots ; \tau_{1}^{(n)}, \ldots, \tau_{\alpha_{n}}^{(n)}\right)}^{\left(q_{1}^{(1)}, \ldots, q_{1}^{(1)}, \ldots q^{(n)}, \ldots, q^{(n)}\right)}$, and consequently the moduli space is covered by the

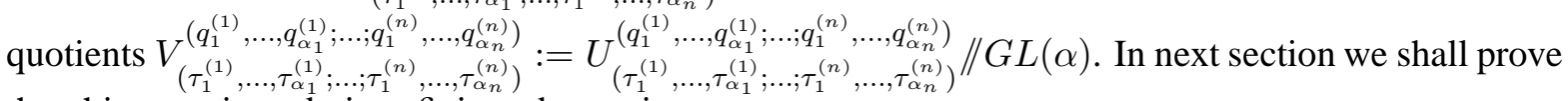
that this covering admits a finite subcovering.

\section{Skeleta OF STABle PAIRS}

Let $Q, \alpha$ and $\zeta$ be as before. Consider a quiver $Q^{\zeta}$ with $Q_{0}^{\zeta}=Q_{0} \cup\{\infty\}$, the arrows of $Q^{\zeta}$ being those of $Q$ together with $\zeta_{i}$ arrows from each $i \in Q_{0}$ to $\infty$. Denote the new arrows by $f_{i q}$, where $i$ indicates the tail of an arrow and $q \in\left\{1, \ldots, \zeta_{i}\right\}$. We also extend the dimension vector $\alpha$ to $\alpha^{\zeta}$, setting $\alpha_{i}^{\zeta}=\alpha_{i}$ for $i=1, \ldots, n$ and $\alpha_{\infty}^{\zeta}=1$.

Observe that the sets $\operatorname{Rep}(Q, \alpha, \zeta)$ and $\operatorname{Rep}\left(Q^{\zeta}, \alpha^{\zeta}\right)$ may be identified in a $\operatorname{GL}(\alpha)$-invariant way. Indeed,

$$
\begin{aligned}
& \operatorname{Rep}(Q, \alpha, \zeta)=\operatorname{Rep}(Q, \alpha) \oplus \bigoplus_{i \in Q_{0}} \operatorname{Hom}_{\mathbb{k}}\left(\mathbb{k}^{\alpha_{i}}, \mathbb{K}^{\zeta_{i}}\right) \cong \\
& \cong \operatorname{Rep}(Q, \alpha) \oplus \bigoplus_{i \in Q_{0}} \operatorname{Hom}_{\mathbb{k}}\left(\mathbb{k}^{\alpha_{i}}, \mathbb{k}\right)^{\zeta_{i}}=\operatorname{Rep}\left(Q^{\zeta}, \alpha^{\zeta}\right) .
\end{aligned}
$$

In terms of matrices this isomorphism has the following interpretation. Let $(M, f)$ be a framed representation and $\widetilde{M}$ be the corresponding representation of $Q^{\zeta}$. Then matrices of $\widetilde{M}_{f_{i q}}, q=1, \ldots, \zeta_{i}$ are rows of the matrix of $f_{i}$ (i.e., what was denoted by $f_{i q}$ in Section 1). This justifies our seeming abuse of notation. 


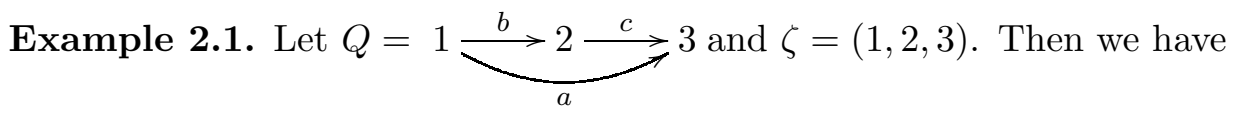

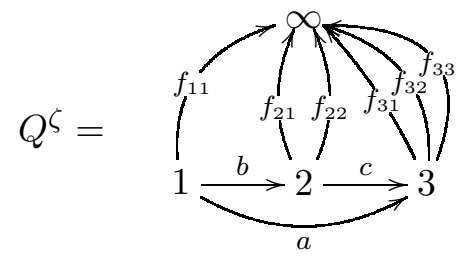

Furthermore, if $\alpha=(2,2,1)$ and

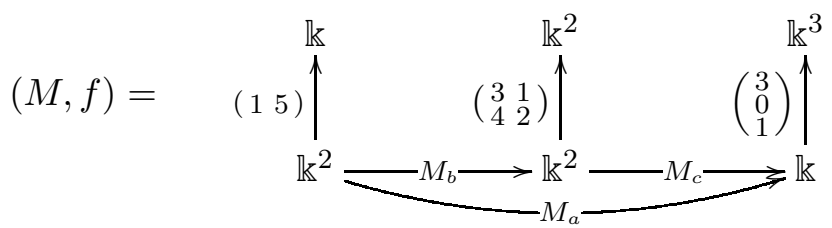

for some $M_{a}, M_{b}$ and $M_{c}$, then the corresponding representation $\widetilde{M}$ of $Q^{\zeta}$ is as follows

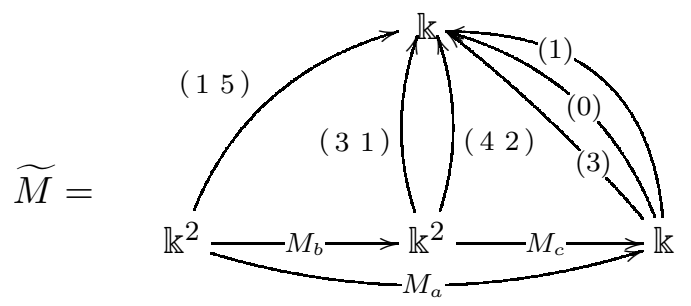

The representation $J$ may also be extended in a natural way to a representation $\widetilde{J}$ of $Q^{\zeta}$. Set $\widetilde{J}_{i}=J_{i}$ and $\widetilde{J}_{a}=J_{a}$, for $i \in Q_{0}$ and $a \in Q_{1}$. Set further $\widetilde{J}_{\infty}=\mathbb{k}$ and $\bigoplus_{b: i \rightarrow \infty} J_{b}: J_{i} \rightarrow \mathbb{k}^{\zeta_{i}}$ be the projection $\prod_{\tau: i \rightsquigarrow j} V_{j}^{(\tau)} \rightarrow V_{i}^{\left(e_{i}\right)}$, for each $i \in Q_{0}$. A straightforward calculation shows that thus constructed $\widetilde{J}$ is isomorphic to the representation $I_{\infty}$ of $Q^{\zeta}$. In particular, elements of $\widetilde{J}_{i}$ may be represented as (possibly infinite) formal series in $\left(f_{j q} \tau\right)^{*}$, for $j \in Q_{0}, q=1, \ldots, \zeta_{j}$, and $\tau: i \rightsquigarrow j$. This implies that paths in $Q^{\zeta}$ may be viewed as linear functions on $\widetilde{J}$.

For a framed representation $(M, f) \in \operatorname{Rep}(Q, \alpha, \zeta)$ consider the corresponding representation $\widetilde{M}$ of $Q^{\zeta}$. The map $\Phi_{(M, f)}$ induces then a morphism $\widetilde{\Phi}_{\widetilde{M}}: \widetilde{M} \rightarrow \widetilde{J}$ of representations of $Q^{\zeta}$ defined by $\widetilde{\varphi}_{i}=\varphi_{i}$, for $i \in Q_{0}, \widetilde{\varphi}_{\infty}=\mathrm{id}_{\mathbb{k}}$. It follows from Corollary 1 that a pair $(M, f)$ is stable if and only if the corresponding map $\widetilde{\Phi}_{\widetilde{M}}$ is an embedding.

Definition. By a $J$-skeleton of a stable pair $(M, f)$ we understand a set $\mathfrak{S}$ of paths of nonzero length in $Q^{\zeta}$ ending in $\infty$ with the following properties:

(1) Restrictions of paths in $\mathfrak{S}$ together with $e_{\infty}$ give a basis in $\widetilde{\Phi}_{\widetilde{M}}(\widetilde{M})^{*}$.

(2) Whenever $\tau a$ is in $\mathfrak{S}$ and $\tau \neq e_{\infty}, \tau$ is also in $\mathfrak{S}$.

The dimension vector of a $J$-skeleton $\mathfrak{S}$ is the dimension vector $\alpha=\underline{\operatorname{dim}}(\mathfrak{S})$ of any stable pair with $J$-skeleton $\mathfrak{S}$. It is easy to see that $\alpha_{i}$ equals the number of paths in $\mathfrak{S}$ ending on $f_{i q}$ for any $q=1, \ldots, \zeta_{i}$. For $i \in Q_{0}$ we set $\mathfrak{S}_{i}=\left\{f_{i q} \tau \in \mathfrak{S}\right\}$.

Lemma 2.2. Every stable pair $(M, f)$ has a J-skeleton.

Proof. Let $(M, f)$ be a stable pair. Let also $\widetilde{M}$ be the corresponding representation of $Q^{\zeta}$. Denote by $N$ its image $\widetilde{\Phi}_{\widetilde{M}}(\widetilde{M}) \subseteq \widetilde{J}$. First of all, we need to show that restrictions of paths 
in $Q$ generate $\widetilde{\Phi}_{\widetilde{M}}(\widetilde{M})^{*}$ as a vector space. Let $\varpi_{1}, \ldots, \varpi_{m}$ be a basis of $\widetilde{\Phi}_{\widetilde{M}}(\widetilde{M})$, where $\varpi_{i}=$ $\sum c_{i q, \tau}\left(f_{i q} \tau\right)^{*}$. Observe that, for some $t$,

$$
\operatorname{dim}\left(\operatorname{span}\left\{\sum_{f_{i_{q}} \tau, l(\tau) \leqslant t} c_{i_{q}, \tau}\left(f_{i q} \tau\right)^{*}\right\}\right)=m,
$$

where $l(\tau)$ stands for the length of a path $\tau$. Then, obviously, the linear span of all paths of length not greater than $t$ generates $\widetilde{\Phi}_{\widetilde{M}}(\widetilde{M})^{*}$.

We see now, that restrictions of paths in $Q$ give a basis of $\Phi_{\widetilde{M}}(\widetilde{M})^{*}$, but less evident is condition (2).

We construct its $J$-skeleton inductively. We start by taking, for each $i \in Q_{0}$, a maximal tuple $f_{i q_{1}}, \ldots, f_{i q_{t}}$ with $\left.f_{i q_{1}}\right|_{N}, \ldots,\left.f_{i q_{t}}\right|_{N}$ linearly independent. Further, on each step we add a path $\tau a$, where $\tau$ is one of the paths we added before, $a$ is an arrow, and $\left.\tau a\right|_{N}$ does not lie in the linear span of restrictions of all the preceding paths. We proceed until we obtain a maximal linearly independent set of restrictions $\Gamma$. It should be proved, however, that it is a basis of $N^{*}$. Let $\left.\tau\right|_{N} \notin \operatorname{span}\{\Gamma\}$. If none of its final subpaths restricted to $N$ is in $\operatorname{span}\{\Gamma\}$, then we have found $f_{l q_{l}}$ whose restriction is not in $\operatorname{span}\{\Gamma\}$, a contradiction. Thus $\tau=\mu \nu$, where $\left.\mu\right|_{N} \in \operatorname{span}\{\Gamma\}$, i.e., $\left.\mu\right|_{N}=\left.\sum_{\kappa \in \Gamma} c_{\kappa} \kappa\right|_{N}$. Consequently, $\left.\tau\right|_{N}=\left.\left.\sum_{\kappa \in \Gamma} c_{\kappa} \kappa\right|_{N} \nu\right|_{N}$. But by maximality of $\Gamma$ each $\left.\left.\kappa\right|_{N} \nu\right|_{N}$ is in $\operatorname{span}\{\Gamma\}$.

Obviously, $\operatorname{Rep}^{s}(Q, \alpha, \zeta)=\bigcup_{\mathfrak{S}} \operatorname{Rep}(Q, \mathfrak{S})$, where

$$
\operatorname{Rep}(Q, \mathfrak{S})=\left\{(M, f) \in \operatorname{Rep}^{s}\left(Q^{\zeta}, \alpha, \zeta\right) \mid(M, f) \text { has } J \text {-skeleton } \mathfrak{S}\right\}
$$

and $\mathfrak{S}$ run through all possible $J$-skeleta for dimension vectors $\alpha$ and $\zeta$. Now note that if $\mathfrak{S}=$ $\left\{f_{1 q_{1}^{(1)}} \tau_{1}^{(1)}, \ldots, f_{q_{1 \alpha_{1}}}^{(1)} \tau_{\alpha_{1}}^{(1)}, \ldots, f_{n q_{1}^{(n)}} \tau_{1}^{(n)}, \ldots, f_{q_{n \alpha_{n}}}^{(n)} \tau_{\alpha_{n}}^{(n)}\right\}$, then $\operatorname{Rep}(Q, \mathfrak{S})$ is exactly the open subset $U_{\left(\tau_{1}^{(1)}, \ldots, \tau_{\alpha_{1}}^{(1)} ; \ldots ; \tau_{1}^{(n)}, \ldots, \tau_{\alpha_{n}}^{(n)}\right)}^{\left(q^{(1)}, \ldots, q^{(1)} ; \ldots ;(n)\right.}$. Observe that there are only finitely many $J$-skeleta, since lengths of paths in an $\alpha$-dimensional $J$-skeleton are bounded by $\max _{i} \alpha_{i}$. Therefore, we obtain a finite covering of $\operatorname{Rep}^{s}(Q, \alpha, \zeta)$ by subsets of form $U_{\left(\tau_{1}^{(1)}, \ldots, \tau_{\alpha_{1}}^{(1)} ; \ldots ; \tau_{1}^{(n)}, \ldots, \tau_{\alpha_{n}}^{(n)}\right)}^{\left(q_{1}^{(1)}, \ldots . q^{(1)} ; \ldots, q^{(n)}, \ldots, q^{(n)}\right)}$.

\section{Embedding of the MOduli SPACE}

Let $\Gamma(\alpha)$ be the set of all paths occurring in $J$-skeleta with dimension vector $\alpha$ and $\widetilde{\Gamma}(\alpha)$ be the union of $\Gamma$ with $\left\{\tau a \mid \tau \in \Gamma(\alpha), a \in Q_{1}, h(a)=t(\tau)\right\}$. Let also $\widehat{J}=\bigoplus_{\tau \in \widetilde{\Gamma}(\alpha)} V_{h(\tau)}^{(\tau)}$. Note that $\widehat{J}$ has a natural $Q_{0}$-grading. Indeed, one may set $\widehat{J}_{i}=\bigoplus_{\tau \in \widetilde{\Gamma}(\alpha), t(\tau)=i} V_{h(\tau)}^{(\tau)}$. Consider the map $\widehat{\Phi}_{(M, f)}$ : $M \rightarrow \widehat{J}$ defined as follows:

$$
\widehat{\varphi}_{i}=\bigoplus_{\tau \in \widetilde{\Gamma}(\alpha)} f_{h(\tau)} \tau: M_{i} \rightarrow \bigoplus_{\tau \in \widetilde{\Gamma}(\alpha), t(\tau)=i} V_{h(\tau)}^{(\tau)}=\widehat{J}_{i} .
$$

It is obvious that a pair $(M, f)$ is stable if and only if $\widehat{\Phi}_{(M, f)}$ is injective. The advantage of $\widehat{\Phi}_{(M, f)}$ is that it maps to a finite dimensional vector space.

We now need to fix notation that will be used throughout the rest of the paper. Let $\widetilde{\Gamma}_{i}(\alpha)$ be a subset of $\widetilde{\Gamma}(\alpha)$ consisting of paths starting at $i$. Let further $\left(B^{(1)}, \ldots, B^{(n)}\right) \in \prod_{i=1}^{n} \operatorname{Mat}_{\left|\widetilde{\Gamma}_{i}(\alpha)\right| \times \alpha_{i}}(\mathbb{k})$. By definition of $\widetilde{\Gamma}(\alpha)$ rows of $B^{(i)}$ correspond to paths in $Q^{\zeta}$ starting at $i$. So, instead of using numerical indices we denote, for a path $\tau$ in $Q$, the row of $B^{(t(\tau))}$ corresponding to $\tau$ by $B_{\tau}$. Let, furthermore, $\mathfrak{T}$ be a subset of $\widetilde{\Gamma}(\alpha)$ (not necessary a skeleton). Denote by $\mathfrak{T}_{i}$ the subset in $\mathfrak{T}$ consisting of paths starting 
at $i$. Set $\underline{\operatorname{dim}}(\mathfrak{T})=\left(\left|\mathfrak{T}_{1}\right|, \ldots,\left|\mathfrak{T}_{n}\right|\right)$. Finally, for a collection $\mathfrak{T}$ with dimensional vector $\alpha$ let $B\left(\mathfrak{T}_{i}\right)$ be the submatrix of $B^{(i)}$ composed of rows corresponding to paths in $\mathfrak{T}_{i}$ and $U(\mathfrak{T})$ be the open subset in $\prod_{i=1}^{n} \operatorname{Mat}_{\left|\widetilde{\Gamma}_{i}(\alpha)\right| \times \alpha_{i}}(\mathbb{k})$ consisting of tuples $\left(B^{(1)}, \ldots, B^{(n)}\right)$ with all $B\left(\mathfrak{T}_{i}\right)$ nondegenerate.

For a $Q_{0}$-graded space $W=\bigoplus_{i \in Q_{0}} W_{i}$ define $\operatorname{IHom}_{\alpha}(W):=\prod_{i \in Q_{0}} \operatorname{IHom}_{\alpha_{i}}\left(W_{i}\right)$, where $\operatorname{IHom}_{\alpha_{i}}\left(W_{i}\right)$ is the set of all injective linear maps from the vector space $\mathbb{k}^{\alpha_{i}}$ to $W_{i}$. We also define $\operatorname{Gr}_{\alpha}(W)$ as the product of Grassmannians $\prod_{i \in Q_{0}} \operatorname{Gr}_{\alpha_{i}}\left(W_{i}\right)$. It is easy to see that $\operatorname{Gr}_{\alpha}(W)$ is a quotient of $\operatorname{IHom}_{\alpha}(W)$ by the natural action of $G L(\alpha)$. We now introduce a map

$$
\widehat{\Phi}: \operatorname{Rep}^{s}(Q, \alpha, \zeta) \rightarrow \operatorname{IHom}_{\alpha}(\widehat{J}), \quad(M, f) \mapsto \widehat{\Phi}_{(M, f)} .
$$

Identify $\operatorname{IHom}_{\alpha}(\widehat{J})$ with an open subset

$$
\bigcup_{\mathfrak{T}: \underline{\operatorname{dim} \mathfrak{T}=\alpha}} U(\mathfrak{T}) \subseteq \prod_{i=1}^{n} \operatorname{Mat}_{\left|\widetilde{\Gamma}_{i}(\alpha)\right| \times \alpha_{i}}(\mathbb{k}) .
$$

It is easy to see that $\operatorname{Im} \widehat{\Phi}$ is contained in

$$
Z(\alpha):=\bigcup_{\substack{\mathfrak{S} \text { is a } J \text {-skeleton } \\ \underline{\operatorname{dim}}(\mathfrak{S})=\alpha}} U(\mathfrak{S}) .
$$

Definition. For a $J$-skeleton $\mathfrak{S}$ set $X(\mathfrak{S})=\operatorname{Im}(\widehat{\Phi}) \cap U(\mathfrak{S})$.

Proposition 3.1. The image of $\widehat{\Phi}$ is a locally closed subvariety in $\operatorname{IHom}_{\alpha}(\widehat{J})$.

Proof. It is sufficient to show that each $X(\mathfrak{S})$ is closed in $U(\mathfrak{S})$. Fix a $J$-skeleton $\mathfrak{S}$. For an arrow $a \in Q_{1}$ set $\mathfrak{S} a=\{\tau a \mid \tau \in \mathfrak{S}, h(a)=t(\tau)\}$. If a tuple of matrices $\left(B^{(1)}, \ldots, B^{(n)}\right) \in$ $U(\mathfrak{S})$ is in $\operatorname{Im} \widehat{\Phi}$, we can recover all the maps $M_{a}, a \in Q_{1}$ of its inverse image $(M, f)$ since for all $a \in Q_{1}: B\left(\mathfrak{S}_{t a} a\right)=B\left(\mathfrak{S}_{t a}\right) M_{a}$ and hence $M_{a}=B\left(\mathfrak{S}_{t a}\right)^{-1} B\left(\mathfrak{S}_{t a} a\right)$. Now observe that $\left(B^{(1)}, \ldots, B^{(n)}\right)$ belongs to the image of $\widehat{\Phi}$ whenever, for all $\tau \in \Gamma(\alpha)$ and $a$ with $t a=h(\tau)$, $B_{\tau a}=B_{\tau} M_{a}$. Using the expression received for $a$, we rewrite this as

$$
B_{\tau a}=B_{\tau} B\left(\mathfrak{S}_{t a}\right)^{-1} B\left(\mathfrak{S}_{t a} a\right) .
$$

Collected together, all these equations define a Zarisky closed subvariety $X(\mathfrak{S})$ in $U(\mathfrak{S})$. Gluing them we obtain a closed subvariety $X_{0}(\alpha) \subseteq Z(\alpha)$ that coincides with $\operatorname{Im} \widehat{\Phi}$. Consequently, $\operatorname{Im} \widehat{\Phi}$ is a locally closed subvariety in $\operatorname{IHom}_{\alpha}(\widehat{J})$.

From the proof of this proposition we infer

Corollary 3.2. The map $\widehat{\Phi}: \operatorname{Rep}^{s}(Q, \alpha, \zeta) \rightarrow \operatorname{Im} \widehat{\Phi}=X_{0}(\alpha)$ is a $G L(\alpha)$-equivariant isomorphism of algebraic varieties.

Proof. To construct the inverse morphism we need to find ways of recovering a pair $(M, f)$ possessing its image $\left(B^{(1)}, \ldots, B^{(n)}\right) \in \operatorname{IHom}_{\alpha}(\widehat{J})$. But $f_{i q}$ comes as $B_{f_{i q}}$ and $M_{a}=$ $B\left(\mathfrak{S}_{t a}\right)^{-1} B\left(\mathfrak{S}_{t a} a\right)$ in $U(\mathfrak{S})$ while on intersections $U(\mathfrak{S}) \cap U(\mathfrak{T})$ the equality $B\left(\mathfrak{S}_{t a}\right)^{-1} B\left(\mathfrak{S}_{t a} a\right)=$ $B\left(\mathfrak{T}_{t a}\right)^{-1} B\left(\mathfrak{T}_{t a} a\right)$ is a direct consequence of the equations defining $X_{0}$.

The equivariance of this isomorphism is obvious.

From now on we will identify each $X(\mathfrak{S}) \subseteq \operatorname{IHom}_{\alpha}(\widehat{J})$ with its preimage $\operatorname{Rep}(Q, \mathfrak{S})=$ $\widehat{\Phi}^{-1}(X(\mathfrak{S})) \subseteq \operatorname{Rep}^{s}(q, \alpha, \zeta)$ and treat it as a subset in $\operatorname{Rep}^{s}(Q, \alpha, \zeta)$ whenever needed. 
Theorem 3.3. In the notation given above

(1) We have

$$
\operatorname{Rep}^{s}(Q, \alpha, \zeta)=\underset{\mathfrak{S} \text { is a J-skeleton }}{\bigcup_{(\mathfrak{S})}}
$$

where $X(\mathfrak{S})$ are Zariski open subsets of $\operatorname{Rep}^{s}(Q, \alpha, \zeta)$ such that $X(\mathfrak{S}) \cong \operatorname{GL}(\alpha) \times \mathbb{A}^{N}$, for some positive integer $N$, and the restriction to $X(\mathfrak{S})$ of the quotient map is the projection onto the second factor. In particular,

$$
\mathcal{M}^{s}(Q, \alpha, \zeta)=\underset{\mathfrak{S} \text { is a J-skeleton }}{\bigcup_{(\mathfrak{S}) / / G L(\alpha)}}
$$

is a covering by open subspaces isomorphic to affine spaces.

(2) The quotient space $\mathcal{M}^{s}(Q, \alpha, \zeta)$ is isomorphic to a locally closed subvariety in $\operatorname{Gr}_{\alpha}(\widehat{J})$.

Proof. Again it will be convenient for us to view $\operatorname{IHom}_{\alpha}(\widehat{J})$ as a Zariski open subset in $\prod_{i \in Q_{0}} \operatorname{Mat}_{\left|\widetilde{\Gamma}_{i}(\alpha)\right| \times \alpha_{i}}(\mathbb{k})$. Fix a $J$-skeleton $\mathfrak{S}$ and assume that its elements are somehow ordered (for example, lexicographically). For a tuple of matrices $B=\left(B^{(1)}, \ldots, B^{(n)}\right)$ define $B_{[\mathfrak{S}]}$ as the $n$-tuple of matrices with $B_{[\mathfrak{S}]}^{(i)}$ a submatrix of $B^{(i)}$ consisting of all the rows of all $B(\mathfrak{S} a)$, for $a \in Q_{1}$ with $t(a)=i$, that do not occur in $B\left(\mathfrak{S}_{i}\right)$. Also denote by $B_{\widehat{\mathfrak{S}}}$ a tuple of matrices with $B_{\widehat{\mathfrak{S}}}^{(i)}$ obtained as a union of $B_{[\mathfrak{S}]}^{(i)}$ with all $B_{f_{i k_{i}}}$, for $f_{i k_{i}} \notin \mathfrak{S}$. Denote by $T_{i}$ the number of rows in $B_{[\mathfrak{S}]}^{(i)}$.

Consider the morphism $\pi_{\mathfrak{S}}: \prod_{i \in Q_{0}} \operatorname{Mat}_{\left|\widetilde{\Gamma}_{i}(\alpha)\right| \times \alpha_{i}}(\mathbb{k}) \rightarrow \prod_{i \in Q_{0}} \operatorname{Mat}_{T_{i} \times \alpha_{i}}(\mathbb{k})$ defined by $B^{(i)} \mapsto$ $\left(B^{(i)} \cdot B\left(\mathfrak{S}_{i}\right)^{-1}\right)_{\widehat{\mathfrak{S}}}$. We claim that it provides the quotient morphism for the action of $G L(\alpha)$ on $U(\mathfrak{S})$.

First of all, $\pi_{\mathfrak{S}}$ is $G L(\alpha)$-invariant, since, for $g \in G L(\alpha)$, we have $\pi_{\mathfrak{S}}(g \cdot B)^{(i)}=$ $\pi_{\mathfrak{S}}\left(B g^{-1}\right)^{(i)}=\left(B^{(i)} g_{i}^{-1} \cdot g_{i} B\left(\mathfrak{S}_{i}\right)^{-1}\right)_{\widehat{\mathfrak{S}}}=\pi_{\mathfrak{S}}\left(B_{i}\right)$. We now prove that $\pi_{\mathfrak{S}}$ is surjective. Let $C \in \prod_{i \in Q_{0}} \operatorname{Mat}_{T_{i} \times \alpha_{i}}(\mathbb{k})$. Recall that each row of each $C^{(i)}$ corresponds to a path from $\bigcup_{a \in Q_{1}}(\mathfrak{S} a \backslash \mathfrak{S}) \cup\left\{f_{i} \notin \mathfrak{S}\right\}$. Take an identity $\alpha_{i} \times \alpha_{i}$-matrix $E^{(i)}$ and put its $j$-th row into correspondence with the $j$-th path from $\mathfrak{S}_{i}$ (with respect to the order we introduced at the beginning of the proof). Now, add to $C^{(i)}$ all the rows of $E^{(i)}$ corresponding to paths from $\mathfrak{S} \cap\left(\bigcup_{a \in Q_{1}, t a=i} \mathfrak{S} a\right)$ and denote the matrix received by $\widetilde{C}^{(i)}$. The first step will be now to recover a stable pair $\left(M^{C}, f^{C}\right)$, then we will use it to obtain a matrix in $\pi_{\mathfrak{S}}^{-1}(C)$. Put $M_{a}^{C}=\widetilde{C}(\mathfrak{S} a)$, for all $a \in Q_{1}$, and

$$
f_{i}^{C}= \begin{cases}E_{f_{i}}^{(i)}, & \text { if } f_{i} \in \mathfrak{S}, \\ C_{f_{i}}^{(i)}, & \text { otherwise. }\end{cases}
$$

Finally, set $B^{C}=\Phi_{\left(M^{C}, f^{C}\right)}$. One should show now that $\pi_{\mathfrak{S}}\left(B^{C}\right)=C$. But $B\left(\mathfrak{S}_{i}\right)=E^{(i)}$, so $B(\mathfrak{S} a)=B(\mathfrak{S}) a=M_{a}=\widetilde{C}(\mathfrak{S} a)$, for all $a \in Q_{1}$, implying that $\pi_{\mathfrak{S}}(B)_{[\mathfrak{S}]}=B_{[\mathfrak{S}]}=C_{[\mathfrak{S}]}$, for $a \in Q_{1}$. Analogously, $B_{f_{i}}^{(i)}=C_{f_{i}}^{(i)}$, for all $f_{i} \notin \mathfrak{S}$. Thus, $\pi_{\mathfrak{S}}(B)=C$ and the surjectivity is proven.

Now we should show that fibers of $\pi_{\mathfrak{S}}$ coincide with $G L(\alpha)$-orbits in $U(\mathfrak{S})$. Observe, that for the above constructed $B^{C} \in \pi_{\mathfrak{S}}^{-1}(C)$ we have $\left(\left(B^{C}\right)^{(i)} \cdot B^{C}\left(\mathfrak{S}_{i}\right)^{-1}\right)(\mathfrak{S})=E^{(i)}$, an identity matrix. But it is easy to see that a $G L(\alpha)$-orbit in $U(\mathfrak{S})$ contains only one tuple of matrices with this property. So, any $B \in \pi_{\mathfrak{S}}^{-1}(C)$ equals $g(B) \cdot B^{C}$, where $g(B)_{i}=B\left(\mathfrak{S}_{i}\right)$. 
An isomorphism GL $(\alpha) \times \prod_{i \in Q_{0}} \operatorname{Mat}_{T_{i} \times \alpha_{i}}(\mathbb{k}) \rightarrow X(\mathfrak{S})$ is therefore obtained by sending a pair $(g, C)$ to $g \cdot B^{C}=\left(\left(B^{C}\right)^{(i)} g_{i}^{-1}\right)_{i \in Q_{0}}$.

To prove the second part it is sufficient to check that each $X(\mathfrak{S}) / / G L(\alpha)$ embeds into $\operatorname{Gr}(\mathfrak{S}):=U(\mathfrak{S}) / / G L(\alpha)$ as a locally closed subvariety. Observe that the natural projection $\pi_{0}: U(\mathfrak{S}) \rightarrow \operatorname{Gr}(\mathfrak{S})$ may be viewed as the map $\prod_{i \in Q_{0}} \operatorname{Mat}_{\left|\widetilde{\Gamma}_{i}(\alpha)\right| \times \alpha_{i}}(\mathbb{k}) \rightarrow$ $\prod_{i \in Q_{0}} \operatorname{Mat}_{\left(\left|\widetilde{\Gamma}_{i}(\alpha)\right|-\alpha_{i}\right) \times \alpha_{i}}(\mathbb{k}), B \mapsto\left(B^{(i)} \cdot B\left(\mathfrak{S}_{i}\right)^{-1}\right)_{\Gamma(\alpha) \backslash \mathfrak{S}}$. Hence $X(\mathfrak{S}) / / G L(\alpha)$ is isomorphic to a subvariety in $\operatorname{Gr}(\mathfrak{S})$ defined by equations

$$
C_{\tau a}=C_{\tau} C\left(\mathfrak{S}_{t a} a\right),
$$

for all paths $\tau$ and arrows $a$ with $\tau a \notin\left(\bigcup_{a \in Q_{1}, t a=i} \mathfrak{S} a\right)$.

Corollary 3.4. In the settings of Theorem 1 we have the following

(1) Each stable pair $(M, f)$ possesses a finite family of normal forms, each normal form corresponding to a $J$-skeleton of $(M, f)$. If $\mathfrak{S}$ is a $J$-skeleton of $(M, f)$, then the respective normal form of $(M, f)$ is $\left(M^{C}, f^{C}\right)$, where $C=\pi_{\mathfrak{S}}(M, f)$.

(2) The following procedure may be used to determine whether two stable pair $(M, f)$ and $\left(M^{\prime}, f^{\prime}\right)$ are isomorphic.

(a) Compute tuples of matrices $B$ and $B^{\prime}$ corresponding to $(M, f)$ and $\left(M^{\prime}, f^{\prime}\right)$.

(b) Find their J-skeleta by seeking non-degenerate maximal minors of $B$ and $B^{\prime}$.

(c) If they have no common J-skeleta, the pairs are not isomorphic.

(d) If $\mathfrak{S}$ a common J-skeleton, compute $\left(M^{C}, f^{C}\right)$ and $\left(M^{C^{\prime}}, f^{C^{\prime}}\right)$ for $C=\pi_{\mathfrak{S}}(B)$ and $C^{\prime}=\pi_{\mathfrak{S}}\left(B^{\prime}\right)$.

(e) The pairs $(M, f)$ and $\left(M^{\prime}, f^{\prime}\right)$ are isomorphic if and only if $\left(M^{C}, f^{C}\right)=$ $\left(M^{C^{\prime}}, f^{C^{\prime}}\right)$.

Remark 3.5. Dimensions of the affine spaces covering the quotient space equal the dimension of the quotient itself, i.e., the difference $\operatorname{dim} \operatorname{Rep}(Q, \alpha, \zeta)-\operatorname{dim} \operatorname{GL}(\alpha)$. For $Q=L_{q, k}$ we have $\operatorname{dim} X(\mathfrak{S}) / / \mathrm{GL}_{m}=\operatorname{dim} \operatorname{Rep}\left(L_{q}, m, k\right)-\operatorname{dim} \mathrm{GL}_{m}=\left(m^{2} q+m k\right)-m^{2}=m(m q+k-m)$, so that $X(\mathfrak{S}) / / G L_{m} \cong \mathbb{A}^{m(m q+k-m)}$.

We have described affine charts covering the quotient. Since all of them are affine spaces, on each we obtain a convenient system of local coordinates. The transition functions between these coordinates may be described in the following way. Let $\mathfrak{S}$ and $\mathfrak{T}$ be two $J$-skeleta, and $B \in$ $\prod_{i \in Q_{0}} \operatorname{Mat}_{\left|\widetilde{\Gamma}_{i}(\alpha)\right| \times \alpha_{i}}(\mathbb{k})$ be a matrix representing a point in $\operatorname{Rep}^{s}(Q, \alpha, \zeta)$. Then we may express $B$ in matrix elements of $\left(B^{(i)} \cdot B\left(\mathfrak{S}_{i}\right)^{-1}\right)_{\widehat{\mathfrak{S}}}$, for $i \in Q_{0}$, and further obtain the expression for $\left(B^{(j)} \cdot B\left(\mathfrak{T}_{j}\right)^{-1}\right)_{\mathfrak{T}}$.

The same procedure may be used to establish relations between normal forms of a stable pair constructed using different $J$-skeleta.

\section{EXAMPLES}

Let $Q$ be the quiver $L_{q}$ and $\alpha=(m)$. It is easy to see that every $J$-skeleton that may occur in this case is a subset of $\left\{f_{i} W\left(a_{1}, \ldots, a_{q}\right) \mid W\right.$ is a word in $a_{j}$ of length at most $\left.m-1\right\}$, so we set

$$
\begin{array}{r}
\widetilde{\Gamma}(m)=\left\{f_{i} W\left(a_{1}, \ldots, a_{q}\right) \mid W \text { is a word in } a_{j} \text { of length at most } m\right\}, \\
\widehat{J}=\bigoplus_{i, W, \text { length }(W) \leqslant m} \mathbb{k} u^{i, W} \text { and } \varphi_{i, W}(m)=f_{i} W\left(a_{1}, \ldots, a_{q}\right) m .
\end{array}
$$


Example 4.1. Let $q=k=1$. This encodes the problem of classifying pairs $(a, f)$, where $a$ is a linear operator on $\mathbb{k}^{m}$ and $f$ is a linear function. The extended quiver $L_{1}^{(1)}=L_{1,1}$ is

$$
a \bigodot 1 \stackrel{f}{\longrightarrow} \infty
$$

There is only one $J$-skeleton, namely $\mathfrak{S}=\left\{f, f a, \ldots, f a^{m-1}\right\}$. Therefore, $\widetilde{\Gamma}(m)=$ $\left\{f, f a, \ldots, f a^{m}\right\}$ and $\widehat{J}=\bigoplus_{i=0}^{m} V_{1}^{\left(f a^{i}\right)}$, where $V_{1}=\mathbb{k}$, so that $\widehat{J}=\mathbb{k}^{m+1}$ as a vector space. Furthermore, we have

$$
\widehat{\Phi}: \operatorname{Rep}\left(L_{1}, m, 1\right) \rightarrow \operatorname{Mat}_{(m+1) \times m}(\mathbb{k}), \quad(a, f) \mapsto \widehat{\Phi}_{(a, f)}=\left(\begin{array}{c}
f \\
f a \\
\vdots \\
f a^{m}
\end{array}\right) .
$$

One can easily see that the stability condition (that is injectivity of $\widehat{\Phi}_{(a, f)}$ ) is in this case equivalent to $f$ being a cyclic vector for the natural action $G L_{m}:\left(\mathbb{k}^{m}\right)^{*}$. The subset $X(\mathfrak{S}) \subseteq$ $\operatorname{Mat}_{(m+1) \times m}(\mathbb{k})$ coincides with $U(\mathfrak{S})$, i.e., consists of matrices $B=\left(b_{i j}\right)$ with first $m$ rows linear independent. Indeed, the only condition $B_{f a^{m}}=B_{f a^{m-1}} B(\mathfrak{S})^{-1} B(\mathfrak{S} a)$ holds identically. Next, for a $(m+1) \times m$-matrix $A$ we have $A_{\widehat{\mathfrak{S}}}=A_{f a^{m}}$, hence the quotient map $\pi_{\mathfrak{S}}$ sends

$$
\pi_{\mathfrak{S}}: B=\left(b_{i j}\right) \mapsto\left(b_{m+1,1}, \ldots, b_{m+1, m}\right)\left(\begin{array}{ccc}
b_{11} & \ldots & b_{1 m} \\
\vdots & & \vdots \\
b_{m 1} & \ldots & b_{m m}
\end{array}\right)^{-1} .
$$

The quotient is isomorphic to $\mathbb{A}^{m}$ and $\pi_{\mathfrak{S}}$ admits a section $\mathbb{A}^{m} \rightarrow \operatorname{Rep}\left(L_{1}, m, 1\right)$ sending $C=\left(c_{0}, \ldots, c_{m-1}\right)$ to the pair

$$
f^{C}=(1,0, \ldots, 0), \quad a^{C}=\left(\begin{array}{ccccc}
0 & 1 & 0 & \ldots & 0 \\
\vdots & \ddots & \ddots & \ddots & \vdots \\
0 & & 0 & 1 & 0 \\
0 & \ldots \ldots & 0 & 1 \\
c_{0} & c_{1} & \ldots & c_{m-2} & c_{m-1}
\end{array}\right)
$$

where $c_{i}$ are the coefficients of the characteristic polynomial of $a$.

Example 4.2. Let $q=1, m=k=2$. The corresponding extended quiver $L_{1}^{(2)}=L_{1,2}$ is

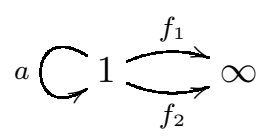

There are three possible $J$-skeleta: $\mathfrak{S}_{1}=\left\{f_{1}, f_{1} a\right\}, \mathfrak{S}_{2}=\left\{f_{2}, f_{2} a\right\}, \mathfrak{S}_{3}=\left\{f_{1}, f_{2}\right\}$. Hence, $\widetilde{\Gamma}(m)=\left\{f_{1}, f_{2}, f_{1} a, f_{2} a, f_{1} a^{2}, f_{2} a^{2}\right\}$, so that

$$
\widehat{\Phi}: \operatorname{Rep}\left(L_{1}, 2,2\right) \rightarrow \operatorname{Mat}_{6 \times 2}(\mathbb{k}), \quad\left(a, f_{1}, f_{2}\right) \mapsto \widehat{\Phi}_{\left(a, f_{1}, f_{2}\right)}=\left(\begin{array}{c}
f_{1} \\
f_{2} \\
f_{1} a \\
f_{2} a \\
f_{1} a^{2} \\
f_{2} a^{2}
\end{array}\right) .
$$

In particular, $\widehat{J}=\mathbb{k}^{6}$. 
First of all we describe the subset $\operatorname{Im} \widehat{\Phi}=X\left(\mathfrak{S}_{1}\right) \cup X\left(\mathfrak{S}_{2}\right) \cup X\left(\mathfrak{S}_{3}\right) \subseteq \operatorname{Mat}_{6 \times 2}(\mathbb{k})$. The chart $X\left(\mathfrak{S}_{1}\right)$ consists of $6 \times 2$-matrices $B=\left(b_{i j}\right)$ with $\left|B\left(\mathfrak{S}_{1}\right)\right|=\left|\begin{array}{ll}b_{11} & b_{12} \\ b_{31} & b_{32}\end{array}\right| \neq 0$ satisfying conditions $B_{f_{2} a}=B_{f_{2}} B\left(\mathfrak{S}_{1}\right)^{-1} B\left(\mathfrak{S}_{1} a\right)$ and $B_{f_{2} a^{2}}=B_{f_{2} a} B\left(\mathfrak{S}_{1}\right)^{-1} B\left(\mathfrak{S}_{1} a\right)$, that may be expanded as

$$
\left(b_{41}, b_{42}\right)=\left(b_{21}, b_{22}\right)\left(\begin{array}{ll}
b_{11} & b_{12} \\
b_{31} & b_{32}
\end{array}\right)^{-1}\left(\begin{array}{ll}
b_{31} & b_{32} \\
b_{51} & b_{52}
\end{array}\right)
$$

and

$$
\left(b_{61}, b_{62}\right)=\left(b_{41}, b_{42}\right)\left(\begin{array}{ll}
b_{11} & b_{12} \\
b_{31} & b_{32}
\end{array}\right)^{-1}\left(\begin{array}{ll}
b_{31} & b_{32} \\
b_{51} & b_{52}
\end{array}\right)
$$

respectively. Similarly we find that $X\left(\mathfrak{S}_{2}\right) \subseteq$ Mat $_{6 \times 2(\mathbb{k})}$ consists of matrices $B=\left(b_{i j}\right)$ with $\left|B\left(\mathfrak{S}_{2}\right)\right|=\left|\begin{array}{ll}b_{21} & b_{22} \\ b_{41} & b_{42}\end{array}\right| \neq 0$ and

$$
\left(\begin{array}{ll}
b_{31} & b_{32} \\
b_{51} & b_{52}
\end{array}\right)=\left(\begin{array}{ll}
b_{11} & b_{12} \\
b_{31} & b_{32}
\end{array}\right)\left(\begin{array}{ll}
b_{21} & b_{22} \\
b_{41} & b_{42}
\end{array}\right)^{-1}\left(\begin{array}{ll}
b_{41} & b_{42} \\
b_{61} & b_{62}
\end{array}\right)
$$

Finally, $X\left(\mathfrak{S}_{3}\right) \subseteq \operatorname{Mat}_{6 \times 2}(\mathbb{k})$ is a subset consisting of matrices $B=\left(b_{i j}\right)$ satisfying $B\left(\mathfrak{S}_{3}\right)=$ $\left|\begin{array}{ll}b_{11} & b_{12} \\ b_{21} & b_{22}\end{array}\right| \neq 0$ and

$$
\left(\begin{array}{ll}
b_{51} & b_{52} \\
b_{61} & b_{62}
\end{array}\right)=\left(\begin{array}{ll}
b_{31} & b_{32} \\
b_{41} & b_{42}
\end{array}\right)\left(\begin{array}{ll}
b_{11} & b_{12} \\
b_{21} & b_{22}
\end{array}\right)^{-1}\left(\begin{array}{ll}
b_{31} & b_{32} \\
b_{41} & b_{42}
\end{array}\right)
$$

Next, we write down the quotient maps $\pi_{\mathfrak{S}_{i}}: \operatorname{Rep}\left(L_{1}, \mathfrak{S}_{i}\right) \cong X\left(\mathfrak{S}_{i}\right) \rightarrow \operatorname{Mat}_{2 \times 2}(\mathbb{k})$;

$$
\begin{gathered}
\pi_{\mathfrak{S}_{1}}:\left(a, f_{1}, f_{2}\right) \mapsto\left(\begin{array}{c}
f_{2} \\
f_{1} a^{2}
\end{array}\right)\left(\begin{array}{c}
f_{1} \\
f_{1} a
\end{array}\right)^{-1}, B=\left(b_{i j}\right) \mapsto\left(\begin{array}{ll}
b_{31} & b_{32} \\
b_{51} & b_{52}
\end{array}\right)\left(\begin{array}{ll}
b_{11} & b_{12} \\
b_{31} & b_{32}
\end{array}\right)^{-1} ; \\
\pi_{\mathfrak{S}_{2}}:\left(a, f_{1}, f_{2}\right) \mapsto\left(\begin{array}{c}
f_{1} \\
f_{2} a^{2}
\end{array}\right)\left(\begin{array}{c}
f_{2} \\
f_{2} a
\end{array}\right)^{-1}, B=\left(b_{i j}\right) \mapsto\left(\begin{array}{ll}
b_{41} & b_{42} \\
b_{61} & b_{62}
\end{array}\right)\left(\begin{array}{ll}
b_{21} & b_{22} \\
b_{41} & b_{42}
\end{array}\right)^{-1} ; \\
\pi_{\mathfrak{S}_{3}}:\left(a, f_{1}, f_{2}\right) \mapsto\left(\begin{array}{c}
f_{1} a \\
f_{2} a
\end{array}\right)\left(\begin{array}{c}
f_{1} \\
f_{2}
\end{array}\right)^{-1}, B=\left(b_{i j}\right) \mapsto\left(\begin{array}{ll}
b_{31} & b_{32} \\
b_{41} & b_{42}
\end{array}\right)\left(\begin{array}{ll}
b_{11} & b_{12} \\
b_{21} & b_{22}
\end{array}\right)^{-1}
\end{gathered}
$$

Each $\pi_{\mathfrak{S}_{i}}$ admits a section $\operatorname{Mat}_{2 \times 2}(\mathbb{k}) \rightarrow \operatorname{Rep}\left(L_{1}, \mathfrak{S}_{i}\right)$ sending $C=\left(x_{a b}^{(i)}\right)$ to the triple $\left(a^{C}, f_{1}^{C}, f_{2}^{C}\right)$ with

$$
\begin{aligned}
& a^{C}=\left(\begin{array}{cc}
0 & 1 \\
x_{21}^{(1)} & x_{22}^{(1)}
\end{array}\right), f_{1}^{C}=(1,0), f_{2}^{C}=\left(x_{11}^{(1)}, x_{12}^{(1)}\right), \text { for } i=1 \\
& a^{C}=\left(\begin{array}{cc}
0 & 1 \\
x_{21}^{(2)} & x_{22}^{(2)}
\end{array}\right), f_{1}^{C}=\left(x_{11}^{(2)}, x_{12}^{(2)}\right), f_{2}^{C}=(1,0), \text { for } i=2 \\
& a^{C}=C=\left(\begin{array}{ll}
x_{11}^{(1)} & x_{12}^{(1)} \\
x_{21}^{(1)} & x_{22}^{(1)}
\end{array}\right), f_{1}^{C}=(1,0), f_{2}^{C}=(0,1), \text { for } i=3 .
\end{aligned}
$$

Now, if a triple $T:=\left(a, f_{1}, f_{2}\right)$ belongs to $\operatorname{Rep}\left(Q, \mathfrak{S}_{i}\right) \cap \operatorname{Rep}\left(Q, \mathfrak{S}_{j}\right)$, for some $i \neq j$, we obtain two normal forms $\left(a^{\pi_{\mathfrak{S}_{i}}(T)}, f_{1}^{\pi_{\mathfrak{S}_{i}}(T)}, f_{2}^{\pi_{\mathfrak{S}_{i}}(T)}\right)$ and $\left(a^{\pi_{\mathfrak{S}_{j}}(T)}, f_{1}^{\pi_{\mathfrak{S}_{j}}(T)}, f_{2}^{\pi_{\mathfrak{S}_{j}}(T)}\right)$ corresponding to $2 \times 2$-matrices $\pi_{\mathfrak{S}_{i}}(T)=\left(x_{a b}^{(i)}\right)$ and $\pi_{\mathfrak{S}_{j}}(T)=\left(x_{a b}^{(j)}\right)$. One easily computes that

$$
\left(\begin{array}{cc}
x_{11}^{(1)} & x_{12}^{(1)} \\
x_{21}^{(1)} & x_{22}^{(1)}
\end{array}\right)=\left(\begin{array}{c}
f_{2} \\
f_{1} a^{2}
\end{array}\right)\left(\begin{array}{c}
f_{1} \\
f_{1} a
\end{array}\right)^{-1}=\left(\begin{array}{cc}
-\frac{x_{11}^{(3)}}{x_{12}^{(3)}} & \frac{1}{x_{12}^{(3)}} \\
x_{12}^{(3)} x_{21}^{(3)}-x_{11}^{(3)} x_{22}^{(3)} & x_{11}^{(3)}+x_{22}^{(3)}
\end{array}\right),
$$




$$
\begin{aligned}
\left(\begin{array}{ll}
x_{11}^{(3)} & x_{12}^{(3)} \\
x_{21}^{(3)} & x_{22}^{(3)}
\end{array}\right)=\left(\begin{array}{cc}
-\frac{x_{11}^{(1)}}{x_{12}^{(1)}} & \frac{1}{x_{12}^{(1)}} \\
\frac{\left(x_{12}^{(1)}\right)^{2} x_{21}^{(1)}-\left(x_{11}^{(1)}\right)^{2}-x_{11}^{(1)} x_{12}^{(1)} x_{22}^{(1)}}{x_{12}^{(1)}} & \frac{x_{11}^{(1)}+x_{12}^{(1)} x_{22}^{(1)}}{x_{12}^{(1)}}
\end{array}\right), \\
\left(\begin{array}{cc}
x_{11}^{(2)} & x_{12}^{(2)} \\
x_{21}^{(2)} & x_{22}^{(2)}
\end{array}\right)=\left(\begin{array}{cc}
-\frac{x_{11}^{(1)}+x_{12}^{(1)} x_{22}^{(1)}}{\left(x_{12}^{(1)}\right)^{2} x_{21}^{(1)}-\left(x_{11}^{(1)}\right)^{2}-x_{11}^{(1)} x_{12}^{(1)} x_{22}^{(1)}} & \frac{x_{12}^{(1)}}{\left(x_{12}^{(1)}\right)^{2} x_{21}^{(1)}-\left(x_{11}^{(1)}\right)^{2}-x_{11}^{(1)} x_{12}^{(1)} x_{22}^{(1)}} \\
x_{21}^{(1)} & x_{22}^{(1)}
\end{array}\right) .
\end{aligned}
$$

We return for a while to the case of arbitrary $m, q$ and $k$. Since the quotient is embedded into $\operatorname{Gr}_{m}(\widehat{J})$, we need to introduce a connection between local coordinates on $Y(\mathfrak{S}):=X(\mathfrak{S}) / / G L(\alpha)$ and Plücker coordinates on $\operatorname{Gr}_{m}(\widehat{J})$. Observe that the latter are of the form $p_{\mathfrak{R}}$, for all subsets $\mathfrak{R} \subseteq \widetilde{\Gamma}(m)$ of cardinality $m$. Indeed, the natural projection $\operatorname{Mat}_{k(m+1)^{q} \times m}(\mathbb{k}) \supseteq \operatorname{IHom}_{m}(\widehat{J}) \rightarrow$ $\operatorname{Gr}_{m}(\widehat{J})$ maps a matrix $B$ to a point $\omega_{B}$, whose Plücker coordinates are $m \times m$-minors of $B$. So, we denote by $p_{\mathfrak{R}}$ the coordinate with corresponding minor consisting of rows prescribed by $\mathfrak{R}$. As for the local coordinates on $Y(\mathfrak{S})$, their definition together with Cramer's rule imply that they are indexed by $m$-element subsets $\mathfrak{R}$ in $\widetilde{\Gamma}(m)$ that may be obtained from $\mathfrak{S}$ by replacement of one of its elements by an element of $(\mathfrak{S} a \backslash \mathfrak{S}) \cup\left\{f_{i} \notin \mathfrak{S}\right\}$.

In Example 4.2, we have $\operatorname{Gr}_{m}(\widehat{J})=\mathrm{Gr}_{2}\left(\mathbb{k}^{6}\right)$. As it was suggested before, we index Plücker coordinates by pairs of paths. For instance, $p_{f_{2}, f_{1} a^{2}}$ stands for $p_{25}$. For a point $\omega_{B}$ corresponding to a rank $m$ matrix $B \in \operatorname{Mat}_{6 \times 2}(\mathbb{k})$ it comes as $\left|\begin{array}{ll}b_{21} & b_{22} \\ b_{51} & b_{52}\end{array}\right|$, and this equals $\left|\begin{array}{c}f_{2} \\ f_{1} a^{2}\end{array}\right|$ if $\left(b_{i j}\right)=$ $\widehat{\Phi}\left(\left(a, f_{1}, f_{2}\right)\right)$. We obtain the expressions

$$
\begin{gathered}
\left(\begin{array}{ll}
x_{11}^{(1)} & x_{12}^{(1)} \\
x_{21}^{(1)} & x_{22}^{(1)}
\end{array}\right)=\left(\begin{array}{cc}
\frac{p_{f_{2}, f_{1} a}}{p_{f_{1}, f_{1} a}} & -\frac{p_{f_{1}, f_{2}}}{p_{f_{1}, f_{1} a}} \\
-\frac{p_{f_{1} a, f_{1} a^{2}}}{p_{f_{1}, f_{1} a}} & \frac{p_{f_{1}, f_{1} a^{2}}}{p_{f_{1}, f_{1} a}}
\end{array}\right), \quad\left(\begin{array}{cc}
x_{11}^{(2)} & x_{12}^{(2)} \\
x_{21}^{(2)} & x_{22}^{(2)}
\end{array}\right)=\left(\begin{array}{cc}
\frac{p_{f_{1}, f_{2} a}}{p_{f_{2}, f_{2} a}} & -\frac{p_{f_{1}, f_{2}}}{p_{f_{2}, f_{2} a}} \\
-\frac{p_{f_{2}} a, f_{2} a^{2}}{p_{f_{2}, f_{2} a}} & \frac{p_{f_{2}, f_{2} a^{2}}}{p_{f_{2}, f_{2} a}}
\end{array}\right), \\
\left(\begin{array}{ll}
x_{11}^{(3)} & x_{12}^{(3)} \\
x_{21}^{(3)} & x_{22}^{(3)}
\end{array}\right)=\left(\begin{array}{cc}
-\frac{p_{f_{2}, f_{1} a} a}{p_{f_{1}, f_{2}}} & \frac{p_{f_{1}, f_{1} a}}{p_{f_{1}, f_{2}}} \\
-\frac{p_{f_{2}, f_{2} a}}{p_{f_{1}, f_{2}}} & \frac{p_{f_{1}, f_{2} a}}{p_{f_{1}, f_{2}}}
\end{array}\right) .
\end{gathered}
$$

Now, we determine the equations in Plücker coordinates that define the closure of the quotient in $\operatorname{Gr}_{2}(\widehat{J})$ in Example 4.2. First of all, there are Plücker relations. We also have the following equations coming from transition relations between $x_{a b}^{(i)}$ and $x_{c d}^{(j)}$

$$
\begin{gathered}
p_{f_{1} a, f_{1} a^{2}} p_{f_{2}, f_{2} a}=p_{f_{2} a, f_{2} a^{2}} p_{f_{1}, f_{1} a}, \quad p_{f_{1}, f_{1} a^{2}} p_{f_{2}, f_{2} a}=p_{f_{2}, f_{2} a^{2}} p_{f_{1}, f_{1} a}, \\
p_{f_{1} a, f_{1} a^{2}} p_{f_{1}, f_{2}}^{2}=p_{f_{1}, f_{1} a}\left(p_{f_{1}, f_{1} a} p_{f_{2}, f_{2} a}-p_{f_{2}, f_{1} a} p_{f_{1}, f_{2} a}\right), \\
p_{f_{2} a, f_{2} a^{2}} p_{f_{1}, f_{2}}^{2}=p_{f_{2}, f_{2} a}\left(p_{f_{1}, f_{1} a} p_{f_{2}, f_{2} a}-p_{f_{2}, f_{1} a} p_{f_{1}, f_{2} a}\right), \\
p_{f_{1}, f_{1} a^{2}} p_{f_{1}, f_{2}}=p_{f_{1}, f_{1} a}\left(p_{f_{1}, f_{2} a}-p_{f_{2}, f_{1} a}\right), \\
p_{f_{2}, f_{2} a^{2}} p_{f_{1}, f_{2}}=p_{f_{2}, f_{2} a}\left(p_{f_{1}, f_{2} a}-p_{f_{2}, f_{1} a}\right) .
\end{gathered}
$$

As we see, not all $p_{\mathfrak{R}}, \mathfrak{R} \subseteq \widetilde{\Gamma}(m)$, occur as numerators of local coordinates on the quotient. Those that do not occur we will call exceed and others essential. We claim that exceed homogeneous coordinates may be eliminated, i.e., that we are able to express them as polynomials in local coordinates in each affine chart. Indeed, we may express $f_{i}$ and $a_{j}$, and then compute all maximal minors of the matrix of $\Phi_{(a, f)}$. For instance, we have

$$
\frac{p_{f_{1} a, f_{2} a}}{p_{f_{1}, f_{1} a}}=\frac{p_{f_{1}, f_{2}} p_{f_{1} a, f_{1} a^{2}}}{p_{f_{1}, f_{1} a^{2}}^{2}}, \quad \frac{p_{f_{1} a, f_{2} a}}{p_{f_{2}, f_{2} a}}=\frac{p_{f_{1}, f_{2}} p_{f_{2} a, f_{2} a^{2}}}{p_{f_{2}, f_{2} a^{2}}^{2}}
$$




$$
\begin{gathered}
\frac{p_{f_{1} a, f_{2} a}}{p_{f_{1}, f_{2}}}=\frac{p_{f_{1}, f_{1} a} p_{f_{2}, f_{2} a}-p_{f_{1}, f_{2} a} p_{f_{1} a, f_{2}}}{p_{f_{1}, f_{2}}^{2}} \\
\frac{p_{f_{1}, f_{2} a^{2}}}{p_{f_{1}, f_{1} a}}=\frac{p_{f_{1}, f_{2}} p_{f_{1} a, f_{1} a^{2}} p_{f_{1}, f_{1} a}+p_{f_{2}, f_{1} a} p_{f_{1}, f_{1} a^{2}} p_{f_{1}, f_{1} a}-p_{f_{1}, f_{2}} p_{f_{1}, f_{1} a^{2}}^{2}}{p_{f_{1}, f_{1} a}^{3}} \\
\frac{p_{f_{1}, f_{2} a^{2}}}{p_{f_{1}, f_{2}}}=\frac{p_{f_{1}, f_{2} a}^{2}-p_{f_{1}, f_{1} a} p_{f_{2}, f_{2} a}}{p_{f_{1}, f_{2}}^{2}} .
\end{gathered}
$$

Returning back to the general case, we may consider an obvious projection-like map $\mathcal{M}^{s}\left(L_{q}, m, k\right) \rightarrow \mathbb{P}^{N}$, where $N+1$ is the number of non-exceed coordinates. Clearly it is well defined. Denote by $\widehat{Y}_{0}$ the image of the quotient in $\mathbb{P}^{N}$. We claim now that the closure $\widehat{Y}$ of $\widehat{Y}_{0}$ (or at least something containing $\widehat{Y}$ as an irreducible component) is defined in $\mathbb{P}^{N}$ solely by the equations that come from transition relations on the quotient, i.e., that Plücker equations are no more required. The reason is that on each affine chart $Y(\mathfrak{S})$, where $\mathfrak{S}$ is a $J$-skeleton, we can express all non-exceed coordinates $p_{\mathfrak{R}}$ in local coordinates of $Y(\mathfrak{S})$ using only this kind of equations, and since $Y(\mathfrak{S})$ is an affine space, these local coordinates are algebraically independent. So, no additional equations are needed.

In the case $q=1, m=k=2$ we have 15 Plücker coordinates, only 9 of them are essential and other 6 are exceed. So, the quotient may be embedded as a locally closed subset into $\mathbb{P}^{8}$.

Example 4.3. Let $q=m=2, k=1$. Denote the loops by $a$ and $b$. There are then two possible $J$-skeleta: $\mathfrak{S}_{1}=\{f, f a\}$ and $\mathfrak{S}_{2}=\{f, f b\}$. Hence $\widetilde{\Gamma}(m)=\left\{f, f a, f a^{2}, f b, f a b, f b a, f b^{2}\right\}$ (we fix this order of paths and construct the map $\widehat{\Phi}$ according to it) and $\widehat{J}=\mathbb{k}^{7}$.

It is not hard to see that

$$
X\left(\mathfrak{S}_{1}\right)=\left\{B \in \operatorname{Mat}_{7 \times 2}(\mathbb{k}) \mid \begin{array}{c}
\left|\begin{array}{cc}
b_{11} & b_{12} \\
b_{21} & b_{22}
\end{array}\right| \neq 0 \\
\left(\begin{array}{ll}
b_{61} & b_{62}
\end{array}\right)=\left(\begin{array}{ll}
b_{31} & b_{32}
\end{array}\right)\left(\begin{array}{ll}
b_{11} & b_{12} \\
b_{21} & b_{22}
\end{array}\right)^{-1}\left(\begin{array}{ll}
b_{21} & b_{22} \\
b_{41} & b_{42}
\end{array}\right) \\
\left(\begin{array}{ll}
b_{71} & b_{72}
\end{array}\right)=\left(\begin{array}{ll}
b_{31} & b_{32}
\end{array}\right)\left(\begin{array}{ll}
b_{11} & b_{12} \\
b_{21} & b_{22}
\end{array}\right)^{-1}\left(\begin{array}{ll}
b_{31} & b_{32} \\
b_{51} & b_{52}
\end{array}\right)
\end{array}\right\}
$$

and

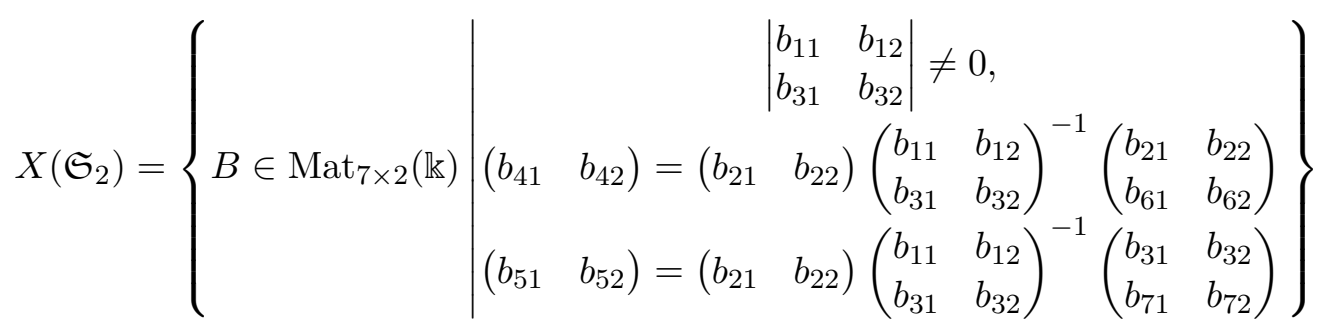

The quotient maps are

$$
\begin{aligned}
& \pi_{\mathfrak{S}_{1}}:(a, b, f) \mapsto\left(\begin{array}{c}
f a^{2} \\
f b \\
f a b
\end{array}\right)\left(\begin{array}{c}
f \\
f a
\end{array}\right)^{-1}, B=\left(b_{i j}\right) \mapsto\left(\begin{array}{ll}
b_{31} & b_{32} \\
b_{41} & b_{42} \\
b_{51} & b_{52}
\end{array}\right)\left(\begin{array}{ll}
b_{11} & b_{12} \\
b_{21} & b_{22}
\end{array}\right)^{-1}, \\
& \pi_{\mathfrak{S}_{2}}:(a, b, f) \mapsto\left(\begin{array}{c}
f a \\
f b a \\
f b^{2}
\end{array}\right)\left(\begin{array}{c}
f \\
f b
\end{array}\right)^{-1}, B=\left(b_{i j}\right) \mapsto\left(\begin{array}{ll}
b_{21} & b_{22} \\
b_{61} & b_{62} \\
b_{71} & b_{72}
\end{array}\right)\left(\begin{array}{ll}
b_{11} & b_{12} \\
b_{31} & b_{32}
\end{array}\right)^{-1},
\end{aligned}
$$


so that $X\left(\mathfrak{S}_{1}\right) / / \mathrm{GL}(\alpha) \cong X\left(\mathfrak{S}_{1}\right) / / \mathrm{GL}(\alpha) \cong \operatorname{Mat}_{3 \times 2}(\mathbb{k}) \cong \mathbb{A}^{6}$. The sections $s_{i}: \operatorname{Mat}_{3 \times 2}(\mathbb{k}) \rightarrow$ $X\left(\mathfrak{S}_{i}\right) \stackrel{\sim}{\rightarrow} \operatorname{Rep}\left(Q, \mathfrak{S}_{i}\right)$ are as follows:

$$
\begin{gathered}
\mathfrak{s}_{1}:\left(\begin{array}{ll}
x_{11}^{(1)} & x_{12}^{(1)} \\
x_{21}^{(1)} & x_{22}^{(1)} \\
x_{31}^{(1)} & x_{32}^{(1)}
\end{array}\right) \mapsto\left(\left(\begin{array}{cc}
0 & 1 \\
x_{11}^{(1)} & x_{12}^{(1)}
\end{array}\right),\left(\begin{array}{cc}
x_{21}^{(1)} & x_{22}^{(1)} \\
x_{31}^{(1)} & x_{32}^{(1)}
\end{array}\right),\left(\begin{array}{ll}
1 & 0
\end{array}\right)\right), \\
\mathfrak{s}_{2}:\left(\begin{array}{ll}
x_{11}^{(2)} & x_{12}^{(2)} \\
x_{21}^{(2)} & x_{22}^{(2)} \\
x_{31}^{(2)} & x_{32}^{(2)}
\end{array}\right) \mapsto\left(\left(\begin{array}{ll}
x_{11}^{(2)} & x_{12}^{(2)} \\
x_{21}^{(2)} & x_{22}^{(2)}
\end{array}\right),\left(\begin{array}{cc}
0 & 1 \\
x_{31}^{(2)} & x_{32}^{(2)}
\end{array}\right),\left(\begin{array}{ll}
1 & 0
\end{array}\right)\right),
\end{gathered}
$$

with transition functions

$$
\left(\begin{array}{cc}
x_{11}^{(1)} & x_{12}^{(1)} \\
x_{21}^{(1)} & x_{22}^{(1)} \\
x_{31}^{(1)} & x_{32}^{(1)}
\end{array}\right)=\left(\begin{array}{cc}
x_{12}^{(2)} x_{21}^{(2)}-x_{11}^{(2)} x_{22}^{(2)} & x_{11}^{(2)}+x_{22}^{(2)} \\
-\frac{x_{11}^{(2)}}{x_{12}^{(2)}} & \frac{1}{x_{12}^{(2)}} \\
\frac{\left(x_{12}^{(2)}\right)^{2} x_{31}^{(2)}-\left(x_{11}^{(2)}\right)^{2}-x_{11}^{(2)} x_{12}^{(2)} x_{32}^{(2)}}{x_{12}^{(2)}} & \frac{x_{11}^{(2)}+x_{12}^{(2)} x_{32}^{(2)}}{x_{12}^{(2)}}
\end{array}\right)
$$

and

$$
\left(\begin{array}{ll}
x_{11}^{(2)} & x_{12}^{(2)} \\
x_{21}^{(2)} & x_{22}^{(2)} \\
x_{31}^{(2)} & x_{32}^{(2)}
\end{array}\right)=\left(\begin{array}{cc}
-\frac{x_{21}^{(1)}}{x_{22}^{(2)}} & \frac{1}{x_{22}^{(1)}} \\
\frac{\left(x_{22}^{(1)}\right)^{2} x_{11}^{(1)}-\left(x_{21}^{(1)}\right)^{2}-x_{12}^{(1)} x_{22}^{(1)} x_{21}^{(1)}}{x_{22}^{(1)}} & \frac{x_{21}^{(1)}+x_{12}^{(1)} x_{22}^{(1)}}{x_{22}^{(1)}} \\
x_{22}^{(1)} x_{31}^{(1)}-x_{21}^{(1)} x_{32}^{(1)} & x_{21}^{(1)}+x_{32}^{(1)}
\end{array}\right)
$$

Having

$$
\left(\begin{array}{ll}
x_{11}^{(1)} & x_{12}^{(1)} \\
x_{21}^{(1)} & x_{22}^{(1)} \\
x_{31}^{(1)} & x_{32}^{(1)}
\end{array}\right)=\left(\begin{array}{cc}
-\frac{p_{f a, f a^{2}}}{p_{f, f a}} & \frac{p_{f, f a^{2}}}{p_{f, f a}} \\
-\frac{p_{f a, f b}}{p_{f, f a}} & \frac{p_{f, f b}}{p_{f, f a}} \\
-\frac{p_{f a, f a b}}{p_{f, f a}} & \frac{p_{f, f a b}}{p_{f, f a}}
\end{array}\right), \quad\left(\begin{array}{ll}
x_{11}^{(2)} & x_{12}^{(2)} \\
x_{21}^{(2)} & x_{22}^{(2)} \\
x_{31}^{(2)} & x_{32}^{(2)}
\end{array}\right)=\left(\begin{array}{cc}
\frac{p_{f a, f b}}{p_{f, f b}} & \frac{p_{f, f a}}{p_{f, f b}} \\
-\frac{p_{f b, f b a}}{p_{f, f b}} & \frac{p_{f, f b a}}{p_{f, f b}} \\
-\frac{p_{f b, f b^{2}}}{p_{f, f b}} & \frac{p_{f, f b^{2}}}{p_{f, f b}}
\end{array}\right),
$$

we obtain the following set of equations

$$
\begin{gathered}
p_{f a, f a^{2}} p_{f, f b}^{2}+p_{f, f a}\left(p_{f a, f b} p_{f, f b a}+p_{f, f a} p_{f b, f b a}\right)=0, \\
p_{f b, f b^{2}} p_{f, f a}^{2}-p_{f, f a}\left(p_{f, f b} p_{f a, f a b}-p_{f a, f b} p_{f, f a b}\right)=0, \\
p_{f, f a^{2}} p_{f, f b}-p_{f, f a}\left(p_{f a, f b}+p_{f, f b a}\right)=0, \\
p_{f, f b^{2}} p_{f, f a}-p_{f, f b}\left(p_{f, f a b}-p_{f a, f b}\right)=0 .
\end{gathered}
$$

So, out of 21 possible coordinates only 11 are essential (i.e., occur as numerators of local coordinates on the quotient) and other 10 ones are exceed. Therefore, the quotient is a locally closed subset in $\mathbb{P}^{10}$ obtained by intersection of nonzero loci of $p_{f, f a}$ and $p_{f, f b}$ with a closed subset defined by the above equations. In particular, $\widehat{Y}$ is a complete intersection. 
FRAMED MODULI SPACES AND TUPLES OF OPERATORS

\section{REFERENCES}

[1] W. Baur, Decidability and undecidability of theories of abelian groups with predicates for subgroups, Compositio Math. 31, 1975, 23-30

[2] J. Engel, M. Reineke, Smooth models of quiver moduli, Math. Z. 262, 2009, 817-848

[3] S. Fedotov, Framed moduli and Grassmannians of submodules, to appear in Trans. Amer. Math. Soc., arXiv:1010.4761 2

[4] B. Huisgen-Zimmermann, Classifying representations by way of Grassmannians, Trans. Amer. Math. Soc. 359, 2007, $2687-2719$

[5] A. D. King, Moduli of representations of finite-dimensional algebras, Quart. J. Math. Oxford Ser. (2) 45(180), 1994, 515-530.

[6] A.I. Kokorin, V.I. Mart'yanov, Universal extended theories, Algebra, Irkutsk., 1973, 107-114

[7] D. Mumford, J. Fogarty, F. Kirwan, Geometric Invariant Theory, Ergebnisse der Mathematik und ihrer Grenzgebiete (2). Springer, Berlin, 1994.

[8] M. Reineke, Framed quiver moduli, cohomology, and quantum groups, J. Algebra 320, 2008, no. 1, 94-115.

Moscow State University, department of Higher Algebra, Russia, 119991, Moscow, GSP-1, 1 Leninskiye Gory, MAIN BUILDING

EMAIL: GLWRATH@YANDEX.RU 